\title{
Empresas B en Latinoamérica: un estudio exploratorio sobre compromiso laboral
}

\author{
Karla Soria-Barreto ${ }^{1}$, Sergio Zuniga-Jara ${ }^{1}$ y Francoise Contreras ${ }^{2}$ \\ (1) Escuela de Ciencias Empresariales, Universidad Católica del Norte, Larrondo 1281, Coquimbo, Chile. \\ (correo-e: ksoria@ucn.cl; sz@ucn.cl) \\ (2) Escuela de Administración, Universidad del Rosario, Calle 200 Autopista Norte, Bogotá, Colombia. \\ (correo-e: francoise.contreras@urosario.edu.co)
}

Recibido Nov. 10, 2020; Aceptado Ene. 13, 2021; Versión final Feb. 10, 2021, Publicado Jun. 2021

\section{Resumen}

En el presente estudio se analiza el compromiso laboral de trabajadores jóvenes y mujeres de las empresas B. Esta es una característica vinculada con el sello distintivo de las empresas B, las que destacan por su preocupación con la comunidad y el medioambiente. Esta investigación exploratoria es la primera basada en información primaria de jóvenes y mujeres que allí trabajan. Se aplicó un instrumento a 264 trabajadores de 16 empresas B de Latinoamérica. Como resultado, el compromiso laboral medio fue $79 \%$, cuatro puntos porcentuales sobre el promedio de América Latina. A nivel de dimensiones del compromiso laboral, "Marca" (reputación) es bien reconocida por sus trabajadores. En cambio, la dimensión "Básicos", sugiere que los beneficios obtenidos (monetarios y no monetarios) por los trabajadores generan bajos niveles de satisfacción. Se concluye que los resultados sugieren avanzar en mejorar los niveles de comunicación interna y en mejorar la forma en que los trabajadores sienten que son partícipes de beneficios, recompensas y reconocimientos.

Palabras clave: empresa B; compromiso laboral; certificación; trabajadores jóvenes; mujeres trabajadoras

\section{B corps in Latin America: an exploratory study on work commitment}

\begin{abstract}
The present study examines work engagement in young employees and women at B corporations. This is a characteristic linked to the distinctive seal of these companies, which stand out for their commitment with the community and the environment. This exploratory research is the first based on first-hand information obtained from young employees and women. An instrument was applied to 264 employees from 16 Latin American B corporations. The results show that the average work commitment was $79 \%$, four percentage points above the Latin America average. At the level of work commitment dimensions, the "Brand" (reputation) is well recognized by employees. In contrast, the results obtained for the "Basic" dimension suggests that obtained benefits (monetary and non-monetary) generate low levels of satisfaction. It is concluded that there is a need to improve both internal communication and the way in which employees feel about how they contribute to their benefits, rewards, and recognitions.
\end{abstract}

Keywords: B Corps; work engagement; certification; young workers; women workers 


\section{INTRODUCCIÓN}

En el año 2006, J.C. Gilbert, B. Houlahan y A. Kassoy desarrollaron en los Estados Unidos una certificación para empresas con triple impacto a través de la organización sin fines de lucro denominada "B Lab" (Pippin y Weber, 2016; Asmus, 2017). Esta Certificación B ("B Corp", "B Corporation" o "Empresas B") está diseñada para empresas que buscan involucrarse con problemas sociales, ambientales y de diversidad laboral, sin que esto impida que logren maximizar sus utilidades (Grimes et al., 2018). Otras denominaciones usuales para referirse a las Empresas B son "Empresas Socialmente Responsables", "Empresas con Propósito" o "Empresas del Cuarto Sector" (Wilburn y Wilburn, 2014; Padilla et al., 2017). Para que las empresas logren y conserven esta certificación deben evaluarse y recibir una calificación mínima de desempeño en cinco dimensiones o áreas de impacto: gobernanza, trabajadores, comunidad, medio ambiente y clientes (Sharma et al., 2018). La empresa también debe permitir auditorías periódicas de certificación y repetir el proceso cada tres años (Conger et al., 2018). El sitio oficial de esta organización (https://bcorporation.net) reporta más de 3.000 empresas certificadas en más de 70 países, mientras que en América Latina existen a la fecha 884 empresas certificadas (sistemab.org) como sigue: Chile 187, Brasil 185, Argentina 131, Colombia 84, México 74, Perú 66, Paraguay 35, Uruguay 35, Ecuador 32, Costa Rica 18, Guatemala 12, Panamá 9, Bolivia 8 y Nicaragua 8.

Existe alguna literatura que ha estudiado a las Empresas $B$, pero hasta ahora se ha hecho fundamentalmente acerca de la motivación de las empresas por obtener la certificación, y acerca del proceso que ello involucra (Muñoz et al., 2018). Actualmente poco se sabe acerca del ámbito laboral al interior de estas empresas, es decir acerca de la perspectiva de los propios trabajadores. En efecto, uno de los ámbitos considerados para lograr la Certificación B es el de los trabajadores, donde se evalúa el desempeño de la empresa respecto de políticas de seguridad laboral, satisfacción laboral, capacitaciones, y beneficios, entre otros. El cumplimiento por parte de una empresa de estos ámbitos laborales lleva a pensar que los trabajadores podrían desarrollar un mayor grado de compromiso laboral, entendido como una gran voluntad para dedicar esfuerzo y concentración al trabajo.

La investigación en las Empresas con Propósito está en sus inicios. La literatura principalmente señala que la certificación es productiva para la sociedad y para las empresas (Muñoz et al., 2018). También se señala que el beneficio social de la certificación es gradual, ya que las Empresas B cambian sus prácticas con el tiempo (Conger et al., 2018 y Sharma et al., 2018). También existe evidencia de que la Certificación B goza de amplio reconocimiento por parte de los propietarios como un "sello de aprobación", lo que les permite validar sus valores organizacionales (Gehman y Grimes, 2017; Grimes et al., 2018). B Lab (2012) realizó un estudio comparativo a nivel mundial entre las empresas tradicionales con Empresas B, encontrando que mientras el $56 \%$ de las Empresas B extienden sus beneficios de salud a los trabajadores a tiempo parcial, solo el $26 \%$ de empresas tradicionales lo hace. Asimismo, el $95 \%$ de las Empresas B posee prácticas de reciclaje, mientras que este porcentaje llega a solo el $26 \%$ en las empresas tradicionales. También que el $35 \%$ de las empresas B tiene como dueñas a mujeres o minorías étnicas frente a un $28 \%$ en empresas tradicionales. A nivel latinoamericano, Muñoz (2015) reporta un análisis para un grupo de 22 Empresas B de Chile, Argentina y Colombia, reportando que los participantes reconocen que el elemento distintivo de las Empresas B es la sostenibilidad, y que existe preocupación de perder esta esencia durante el proceso de crecimiento. Por otro lado, las empresas $B$ parecen favorecer la heterogeneidad de género, de edad y de grupo étnico en sus trabajadores, entre otros factores (Salanova y Schaufeli, 2004).

En este estudio estamos interesados en analizar una dimensión diferente de estas empresas en Latinoamérica, ya que un grupo de trabajadores de especial interés son los jóvenes y las mujeres, quienes se han caracterizado por una mayor dificultad para ser incluidos en el mercado laboral. Actualmente en América Latina existen más de 30 millones de personas, en su mayoría jóvenes y mujeres, los que corren el riesgo de volver a la pobreza (UNDP, Human Development Report 2016). Existe aquí un importante número de empleos precarios e informales en los que se emplean principalmente a mujeres y jóvenes. Más de la mitad de los 300 millones de trabajadores de América Latina y el Caribe trabajan en empleos informales. El $70 \%$ de las PYME son informales, y las microempresas son las principales empleadoras (Ministerio de Economía, Fomento y Turismo, 2018). Es por ello que en esta investigación se busca entender mejor los impactos de las Empresas $B$ respecto de la inclusión de mujeres y jóvenes. Se busca entender cómo estas empresas generan mayores y mejores oportunidades sociales y económicas para estos grupos en América Latina.

Por otra parte, desde el punto de vista de trabajador, una Empresa B debiera estar enfocada no solamente en el resultado económico, sino también en sus trabajadores, y por ello debieran existir mayor preocupación por generar un clima laboral positivo (da Costa et al., 2014). Esto se debiera traducir en mayores niveles de compromiso laboral. Esta es la hipótesis central de este estudio, es decir, nuestro objetivo es verificar si el nivel de compromiso de trabajadores jóvenes y mujeres de las Empresas $B$ de Latinoamérica se encuentra por encima del promedio de las demás empresas de la región. Es decir, se busca verificar si una Empresa $B$ logra transmitir a sus empleados jóvenes y mujeres sus prácticas distintivas. Se trata de un estudio de tipo 
exploratorio, que busca proveer lineamientos a futuras investigaciones, potenciar la Certificación B, y definir políticas para enfrentar las restricciones de jóvenes y mujeres para insertarse al mercado laboral.

\section{OTROS ANTECEDENTES}

El compromiso laboral es un estado psicológico de los empleados, el que tiene implicancias directas sobre la organización (Sinval et al. 2018). Empleados comprometidos suelen ser más seguros, sanos y dispuestos a realizar mayores esfuerzos (Fleming y Asplund, 2007). A mayor nivel de compromiso por parte de los trabajadores, se espera que los resultados globales de esa empresa mejoren. El compromiso de los empleados parece ser la antítesis positiva del agotamiento laboral, contribuyendo de manera positiva a la salud y bienestar de las personas (Maslach et al., 2001). El compromiso puede incluirse dentro del concepto de Engagement, un estado persistente y positivo de compromiso afectivo-emocional con empleados que experimentan vigor, dedicación y absorción (inmersión total) en su trabajo (Salanova y Schaufeli, 2004; Schaufeli y Baker, 2010).

Los empleados extremadamente comprometidos realizan su labor en pro de la organización (Albdour y Altarawneh, 2014). Los trabajadores comprometidos presentan un estado cognitivo, emocional y conductual dirigido hacia los resultados organizacionales deseados (Shuck y Wollard, 2010). Las empresas que tienen una fuerza laboral más comprometida tienen una reputación más favorable por sus aspectos sociales y su responsabilidad medioambiental (Brammer et al., 2007, y Ali et al., 2010). Estas organizaciones se han vuelto más atractivas para empleados potenciales, lo que podría conllevar, dependiendo del grado de colaboración de los empleados, a propagar las actividades y políticas sociales de la organización como también a que se implementen en ellas, comportamientos éticos (Collier y Esteban, 2007).

Un contexto laboral de alto compromiso es más acorde con las expectativas laborales de las generaciones más jóvenes, en las que es fundamental el equilibrio trabajo-vida personal. Los empleados esperan ser remunerados de manera justa y equitativa, con mayores posibilidades de desarrollo profesional para avanzar de forma rápida con mejoras salariales. Sin embargo, se trata de una generación que busca algo más de su trabajo que solo la retribución, y esperan que sea significativo, estimulante y desafiante, es decir, que sea fuente de satisfacción. Asimismo, las generaciones jóvenes desean pertenecer a una organización que valore la importancia de ser responsable socialmente, y se les entregue la oportunidad de ampliar sus horizontes con la posibilidad de experiencias de movilidad ( $\mathrm{Ng}$ et al., 2010).

Por otro lado, las organizaciones modernas esperan que los empleados sean proactivos, tengan iniciativa personal, que colaboren entre ellos y que tengan un compromiso con la excelencia. Si esto se logra, las empresas pueden alcanzar bajos índices de ausentismo (Salanova y Schaufeli, 2004). Por lo tanto, las organizaciones desarrollan estrategias para atraer y motivar a los empleados en un nuevo entorno económico y dinámico. Otra característica actual, es que se hace necesario renovar y promover estudios en los trabajadores jóvenes, dado que el desempleo y la precariedad laboral inciden con especial intensidad en los menores de 30 años (Martínez-Rodríguez y Carmona, 2010). En resumen, en las Empresas B, dadas sus buenas prácticas, el mayor nivel de inclusión laboral debiera reflejarse en el nivel de compromiso laboral de sus trabajadores, por lo cual se esperaría que éstas estuvieran por encima del promedio de otras organizaciones. Para medir empíricamente el nivel de compromiso laboral la forma más difundida incluye seis dimensiones (ver Tabla 1). Las Empresas B, dadas sus buenas prácticas, debieran reflejar mayores niveles de compromiso laboral de sus trabajadores, junto a una menor rotación y ausentismo. Como resultado, además de empleados más productivos, se lograrían clientes con mayor satisfacción y mejores ingresos y rentabilidad para la empresa (Mehech et al., 2016).

En esta investigación, que es una de las primeras en que se analiza información primaria de los trabajadores de Empresas B en Latinoamérica, se obtuvo información de interés acerca de la percepción de los trabajadores respecto de la reputación de las empresas, diversidad e inclusión, seguridad laboral, aprendizaje y desarrollo, entre otras. Esta investigación exploratoria hace un aporte en avanzar en cubrir la brecha de conocimiento actual de las Empresas B en América Latina. A continuación se realizan mediciones muestrales del nivel de compromiso de trabajadores jóvenes y mujeres en Empresas $B$ de Latinoamérica, y se compara el nivel promedio con el de las demás empresas de la región. Para ello, en el siguiente apartado se describe la metodología y la recopilación de los datos. Luego se reportan los resultados, y se finaliza con el apartado de conclusiones.

\section{METODOLOGÍA}

Para realizar el diagnóstico del estado del compromiso laboral de los trabajadores jóvenes (18-24 años) y mujeres de las Empresas B de Latinoamérica, se elaboró un cuestionario de tres partes. En la primera se pidió información de la empresa: sector económico, antigüedad de la empresa (años); antigüedad como empresa B (años), número de empleados totales y de mujeres y jóvenes. Este fue respondido por el 
propietario, directivo y/o encargado de Recursos Humanos de la organización (RRHH). La segunda parte recoge información personal y de la familia de cada trabajador joven y mujer, incluyendo datos de género, edad, nivel educacional, estado civil, salario, antigüedad en la empresa, unidad (área) de trabajo, cargo, entre otras.

La tercera parte levanta información sobre el compromiso laboral basados en un modelo que ya ha sido aplicado en varios países (Mehech et al., 2016, basados en Aon Hewitt, 2016, 2017), lo que permite la comparabilidad de los resultados. El cuestionario consta de 23 preguntas formuladas en una escala de Likert de 5 categorías de acuerdo-desacuerdo, desde 1 (totalmente en desacuerdo) hasta 5 (totalmente de acuerdo). Respecto del grado de satisfacción del trabajador se evalúan 6 dimensiones: 1) el sello de la empresa ("Marca"); 2) la gestión de la empresa ("Liderazgo"); 3) las oportunidades y trato que recibe el empleado dentro de ella ("Desempeño"); 4) la vinculación de la empresa con el entorno, diversidad e inclusión ("Prácticas"); 5) la capacitación y autonomía del empleado y la comunicación interna de la organización ("Trabajo"); y finalmente 6) los beneficios básicos que ofrece la empresa al trabajador, como estabilidad laboral, seguridad en el trabajo, ambiente de trabajo, balance personal y laboral, y remuneración ("Básicos"). La Tabla 1 (Mehech et al., 2016, p. 52, basado en Aon Hewitt, 2016 y 2017), muestra las dimensiones y las preguntas.

Tabla 1: Compromiso Laboral: Dimensiones y Subdimensiones

\begin{tabular}{|c|c|c|}
\hline Dimensiones & Subdimensiones & Pregunta \\
\hline \multirow[t]{3}{*}{ 1. Marca } & Reputación & Siento orgullo de trabajar en una empresa de alta reputación. \\
\hline & $\begin{array}{l}\text { Propuesta de Valor al } \\
\text { Empleado }\end{array}$ & Estoy contento por como la empresa en la que trabajo me valora. \\
\hline & $\begin{array}{l}\text { Responsabilidad } \\
\text { Empresarial }\end{array}$ & $\begin{array}{l}\text { Estoy orgulloso de la conciencia social y ecológica empresarial que tiene la empresa } \\
\text { en la que trabajo. }\end{array}$ \\
\hline \multirow[t]{2}{*}{ 2. Liderazgo } & Alta Dirección & Me siento a gusto con la alta dirección y su manejo de la empresa. \\
\hline & Unidades de Negocios & Estoy satisfecho de cómo mis jefes directos me involucran en el trabajo. \\
\hline \multirow[t]{6}{*}{ 3. Desempeño } & $\begin{array}{l}\text { Oportunidades de } \\
\text { Carrera }\end{array}$ & Me motiva saber que puedo crecer profesionalmente dentro de la empresa. \\
\hline & $\begin{array}{l}\text { Aprendizaje y } \\
\text { Desarrollo }\end{array}$ & En mi trabajo aprendo y desarrollo nuevas capacidades. \\
\hline & Gestión Desempeño & Estoy de acuerdo de cómo se valora mi desempeño dentro de la empresa. \\
\hline & Gestión Personas & Siento que en mi empresa hay un buen manejo de las personas. \\
\hline & Reconocimiento & Siento que mi trabajo es reconocido por mis compañeros y jefes. \\
\hline & Recompensa & Me siento conforme con que la recompensa de mi trabajo es acorde a lo que hago. \\
\hline \multirow[t]{4}{*}{ 4. Prácticas } & Comunicación Externa & $\begin{array}{l}\text { Me gusta la capacidad que tiene la empresa para comunicarse con entidades } \\
\text { externas. }\end{array}$ \\
\hline & Orientación Cliente & Me siento orgulloso de como mi empresa le da importancia a los clientes. \\
\hline & Diversidad e Inclusión & Me gusta que mi empresa se preocupe de la diversidad e inclusión cultural. \\
\hline & Infraestructura & Puedo realizar mi trabajo de manera cómoda, gracias a las instalaciones existentes. \\
\hline \multirow[t]{5}{*}{ 5. Trabajo } & Colaboración & $\begin{array}{l}\text { Me siento feliz de la relación que existe con mis compañeros y el apoyo que me } \\
\text { brindan. }\end{array}$ \\
\hline & Capacitación & Estoy satisfecho con las capacitaciones que entrega la empresa. \\
\hline & Autonomía & Puedo realizar mi trabajo de manera autónoma. \\
\hline & Tareas Laborales & $\begin{array}{l}\text { Siento que las tareas que me entregan están alineadas con los objetivos de la } \\
\text { empresa. }\end{array}$ \\
\hline & Comunicación Interna & $\begin{array}{l}\text { Siento que la compañía maneja muy bien la comunicación entre sus áreas, entre } \\
\text { supervisor y supervisado, y entre pares. }\end{array}$ \\
\hline \multirow[t]{3}{*}{ 6. Básicos } & Beneficios & $\begin{array}{l}\text { Me siento satisfecho con los beneficios que obtengo, tanto monetarios como no } \\
\text { monetarios. }\end{array}$ \\
\hline & Estabilidad Laboral & Me siento seguro en mi trabajo ya que me parece un trabajo estable. \\
\hline & Seguridad Laboral & Estoy tranquilo por trabajar en un ambiente seguro. \\
\hline
\end{tabular}

Un enfoque alternativo de medición es el modelo UWES (Escala Utrecht de Engagement en el Trabajo de Schaufeli y Bakker, 2010) de tres variables, que incluye el vigor, la dedicación y la absorción de los empleados en el trabajo. Sin embargo, se considera que el modelo de seis variables (Tabla 1) puede ser más preciso como herramienta de medición de la generación de compromiso en los empleados. Esto debido a que se diferencia dos grupos: las variables de Fundación y las variables Diferenciadoras. En el grupo Fundación se incluye variables básicas o estructurales del compromiso: "Prácticas" de la empresa, el "Trabajo" y los "Básicos". Respecto del grupo Diferenciador, aparecen variables que marcan la diferencia entre un nivel bueno de compromiso y un nivel superlativo: "Marca", "Liderazgo" y "Desempeño".

\section{RESULTADOS}

Para aplicar el cuestionario de la Tabla 1, "Sistema B" (organización representante de B Lab en Latinoamérica) envió 541 correos electrónicos invitando a las Empresas B de América Central, Argentina, Brasil, Chile, 
Colombia, México, Paraguay, Perú y Uruguay para invitarlas a ser parte de este estudio. Como resultado de esta invitación, 54 empresas se interesaron y fueron contactadas, pero de ellas finalmente 16 empresas de siete países latinoamericanos efectivamente participaron del estudio. Todo el proceso de recopilación de información se realizó durante los meses de abril y mayo del año 2018.

La Tabla 2 muestra el detalle de la información primaria recogida, con un total de 264 cuestionarios respondidos por trabajadores jóvenes y/o mujeres. Respecto de la información demográfica básica, la mayor cantidad de trabajadores encuestados tiene entre 25-34 años (42\%). Las labores que desempeñan son asistentes $(47 \%)$, cargos de jefatura (25\%), operarios (18\%) y gerentes (10\%). Más del $50 \%$ de los encuestados señaló tener a lo menos 8 años de antigüedad en la empresa. La mayoría de los encuestados son mujeres (242), de las cuales 43 son jóvenes, mientras que sólo hay 22 jóvenes hombres. En resumen, la Tabla 2 caracteriza bastante bien la presencia de ambos grupos objetivo en las empresas analizadas.

Tabla 2: Número de Empresas Encuestadas y Tasa de Respuesta

\begin{tabular}{|l|c|c|c|c|c|c|}
\hline & \multirow{2}{*}{$\begin{array}{c}\text { Número } \\
\text { de Empresas }\end{array}$} & \multicolumn{3}{|c|}{ Número Trabajadores } & \multicolumn{2}{c|}{ Tasa de Respuesta } \\
\cline { 3 - 7 } & & Jóvenes & Mujeres & Total & Jóvenes & Mujeres \\
\hline Brasil & 2 & 1 & 10 & 11 & $25.6 \%$ & $32.4 \%$ \\
\hline Chile & 3 & 4 & 33 & 37 & $50.8 \%$ & $57.1 \%$ \\
\hline Colombia & 5 & 32 & 155 & 167 & $19.3 \%$ & $24.4 \%$ \\
\hline México & 2 & 2 & 2 & 4 & $25.0 \%$ & $100.0 \%$ \\
\hline Paraguay & 1 & 1 & 1 & 1 & $9.1 \%$ & $100.0 \%$ \\
\hline Perú & 2 & 12 & 31 & 33 & $67.4 \%$ & $120.0 \%$ \\
\hline Uruguay & 1 & 3 & 10 & 11 & $31.3 \%$ & $75.0 \%$ \\
\hline Total/Promedio & 16 & 65 & 242 & 264 & $24.1 \%$ & $34.4 \%$ \\
\hline
\end{tabular}

\section{Compromiso laboral: comparación internacional}

Las respuestas de compromiso basadas en la escala de Likert de 5 categorías fueron tabuladas como sigue: Totalmente en Desacuerdo=1; En Desacuerdo=2; Ni de Acuerdo Ni en Desacuerdo=3; De Acuerdo=4; Totalmente de Acuerdo=5. Si una dimensión tiene tres subdimensiones, el máximo puntaje que puede alcanzar por empleado para esa dimensión es 15 puntos, y si un empleado le asignó a esa dimensión 10 puntos, el porcentaje asociado a la dimensión es de $66,7 \%$. Esto es equivalente a asignar valores de $20 \%$ para 1 (totalmente en desacuerdo) hasta 100\% para 5 (totalmente de acuerdo) y luego agrupar por dimensiones, empresas y países como promedios aritméticos de las respuestas. La Tabla 3 muestra los niveles de compromiso promedio observado en las empresas, por países. Se reporta un promedio global del $79 \%$. Llama la atención que el menor nivel promedio es de Chile, mientras que los trabajadores de Uruguay reportaron los mayores niveles.

Tabla 3: Estadísticas Descriptivas de Compromiso Laboral: mujeres y jóvenes

\begin{tabular}{|l|c|c|c|c|c|}
\hline País & Num. de Obs. & Promedio & Desv. St. & Mínimo & Máximo \\
\hline Brasil & 11 & $81.7 \%$ & 0.0846 & $62.9 \%$ & $93.5 \%$ \\
\hline Chile & 37 & $70.6 \%$ & 0.2329 & $20.0 \%$ & $100.0 \%$ \\
\hline Colombia & 167 & $79.6 \%$ & 0.2062 & $20.0 \%$ & $100.0 \%$ \\
\hline México & 4 & $82.2 \%$ & 0.1042 & $72.1 \%$ & $94.2 \%$ \\
\hline Paraguay & 1 & $84.0 \%$ & & $84.0 \%$ & $84.0 \%$ \\
\hline Perú & 33 & $82.1 \%$ & 0.0928 & $56.0 \%$ & $98.3 \%$ \\
\hline Uruguay & 11 & $85.0 \%$ & 0.0831 & $74.2 \%$ & $100.0 \%$ \\
\hline Total & 264 & $79.0 \%$ & 0.1931 & $20.0 \%$ & $100.0 \%$ \\
\hline
\end{tabular}

Globalmente estos resultados se pueden comparar con el Informe de Compromiso Laboral del año 2017 para América Latina de la consultora Aon Hewitt (2018), cuyo promedio fue del $75 \%$, con datos del año 2017 , excepto Perú con datos de 2014. La Figura 1 muestra comparativamente los resultados de este estudio para jóvenes y mujeres, comparados con el reporte de Aon Hewitt (2017). Se aprecia que en casi todos los países (sin datos para Paraguay y Uruguay), el nivel de compromiso laboral medido para las Empresas B superó el promedio de su respectivo país. Esto, con excepción de Colombia, donde se observa justamente lo opuesto. Esto sugiere como resultado de este análisis exploratorio, que los trabajadores de las Empresas B declararon 4 puntos porcentuales más de compromiso laboral que el promedio de América Latina. 


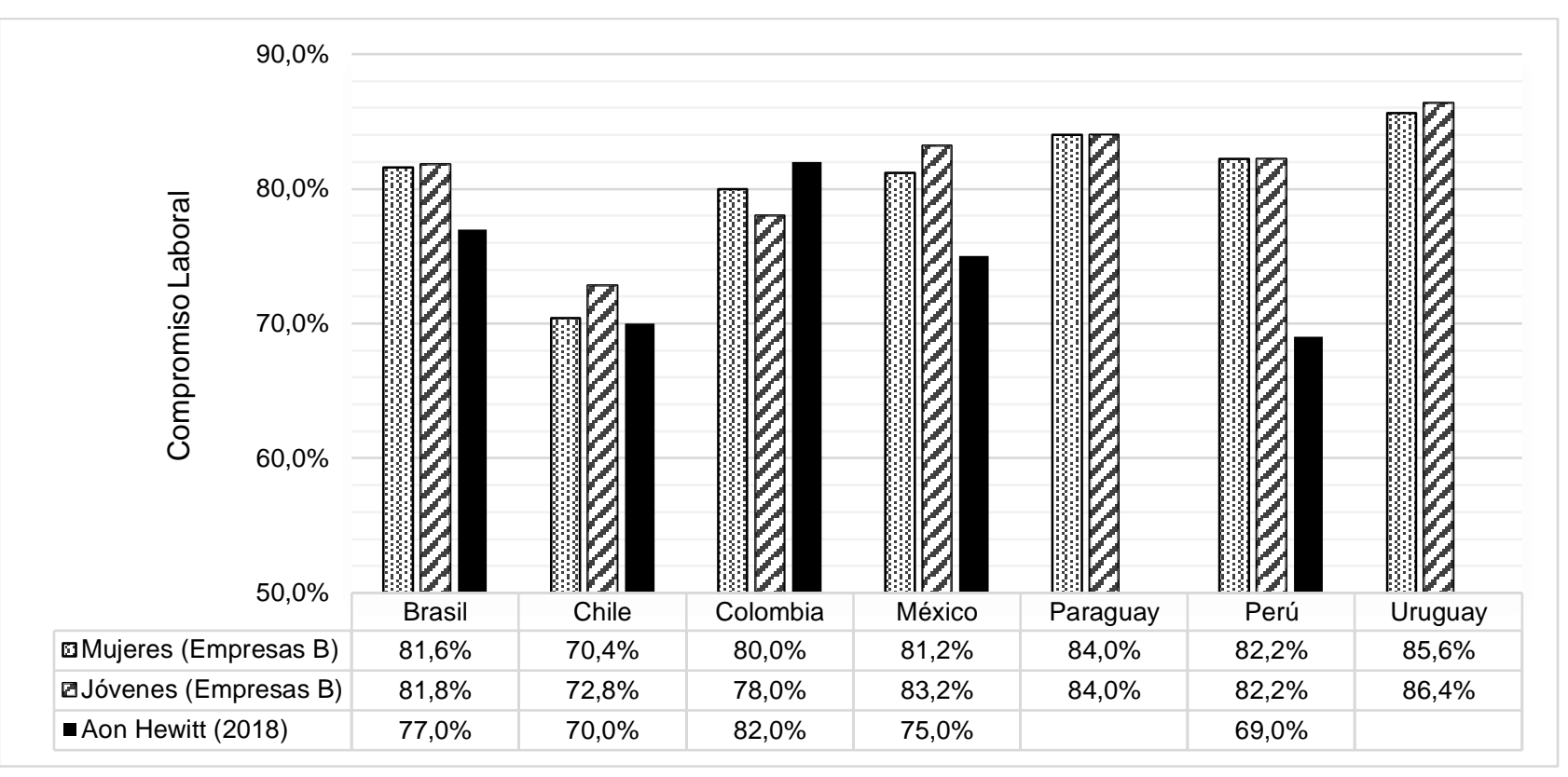

Fig. 1: Compromiso Laboral de trabajadores de Empresas B respecto de otras empresas

El nivel de compromiso encontrado para las Empresas B partícipes de este estudio fue de un $79 \%$, cuatro puntos porcentuales sobre el promedio para América Latina, ya que la media de Brasil, Chile, Colombia, México y Perú es de $74.6 \%$ en la Figura 1 para la fila Aon Hewitt (2018). Es importante notar que la diferencia se explica fundamentalmente por los bajos resultados reportados por el Perú y Chile, y que si se excluye Perú (que tiene datos de un año distinto), entonces la diferencia es de solo tres puntos porcentuales.

En el caso de las Empresa B el promedio de compromiso laboral de las mujeres, que llega a $79,2 \%$, es levemente superior al de los hombres, cuyo promedio es de 76,4\%. Además, en las Empresas B estudiadas se observó una diferencia entre los niveles de compromiso por edad: las trabajadoras con mayor edad (4554 años) son quienes muestran un mayor nivel de compromiso laboral con casi $90 \%$ (89.2\%). Le siguen tres rangos de edad: los trabajadores entre 18-24 años (jóvenes), 35-44 años y 54-65 años son quienes muestran un segundo nivel de compromiso laboral, con un promedio de $81.2 \%$. Finalmente, el tercer grupo de trabajadoras con edades entre 25-34 años, muestran el menor nivel de promedio de compromiso laboral con un $75.8 \%$.

\section{Dimensiones del compromiso laboral: Recomendaciones}

En principio, se espera que las dimensiones de compromiso "Marca" y "Básicos" sean las más valoradas por los jóvenes y mujeres que trabajan en las Empresas B, dadas las características de estas empresas (Muñoz, 2015). En efecto, todos sus componentes tienen directa relación con la certificación de Empresas B. Por ejemplo, "Marca" incluye la valoración de la reputación de la empresa y la responsabilidad empresarial, mientras que en "Básicos" se incorporan preguntas referidas a beneficios, estabilidad y seguridad laboral. Por otro lado, la dimensión con menores características de las Empresas B es la relacionada con "Liderazgo", ya que este aspecto se atribuye a cualquier organización. En cambio, las dimensiones de "Desempeño", "Prácticas" y "Trabajo" comparten características que se espera estén presentes en ambos tipos de empresas. En la Figura 2 se muestran en el eje horizontal las dimensiones que se espera tengan mayor (a la izquierda) a menor (a la derecha) relación con el espíritu de las Empresas de Triple Impacto. En el eje vertical se muestran los niveles de compromiso laboral promedio observados. Se aprecia que la dimensión con mayor valoración fue "Marca" (82.1\%), tal como se esperaba, sugiriendo que los trabajadores sienten orgullo de trabajar en una empresa de alta reputación, conciencia social y ecológica de su empresa (Padilla et al., 2017). La siguiente dimensión es "Básicos", que sin embargo logra un valor promedio de sólo $78.7 \%$, algo por debajo del promedio global (79\%, indicado con una línea horizontal). Aquí los trabajadores parecen mostrarse menos satisfechos con los beneficios que obtienen, sugiriendo que las empresas B pueden mejorar el grado de identificación de sus trabajadores con los resultados monetarios como no monetarios de estas empresas. Sin embargo, sí es valorado el trabajar con estabilidad y seguridad laboral. Respecto de la dimensión "Desempeño", se observa aquí el nivel de menor valoración (75.7\%), reflejando que los trabajadores se sienten poco conformes con la recompensa por el trabajo realizado, y que no sienten que su trabajo es realmente reconocido por compañeros y jefes. En la dimensión "Prácticas", los resultados son bastante satisfactorios (81.9\%), superando ampliamente el promedio global. Los trabajadores declaran identificarse con la preocupación respecto de la diversidad e inclusión cultural. También valoran las comodidades existentes para realizar el trabajo y se sienten orgullosos de la forma en que se trata a los clientes. 
Finalmente, respecto de "Trabajo", los empleados jóvenes y mujeres declaran no estar plenamente satisfechos con las capacitaciones que entrega la empresa. Además, sienten que la empresa debe mejorar la forma en que la empresa maneja las comunicaciones entre sus áreas, entre supervisor y supervisado, y entre pares, ya que esta es la pregunta que recibió la calificación más baja. Respecto de "Liderazgo", una dimensión que no es característica de estas empresas, se da menor valoración a la forma en que sus jefes los involucran en el trabajo.

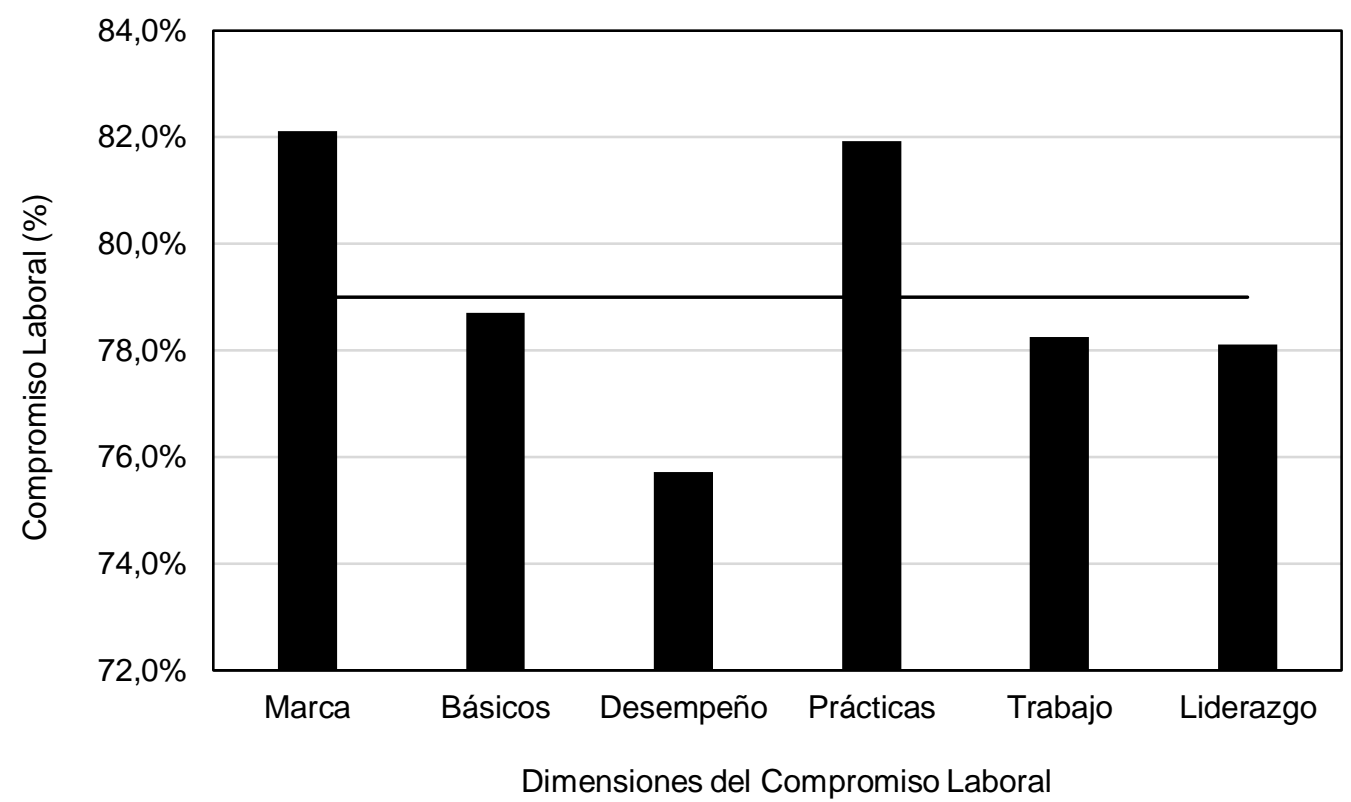

Fig. 2: Niveles de compromiso de los trabajadores jóvenes y mujeres de Empresas B en Latinoamérica, por Dimensión

\section{CONCLUSIONES}

1.- Esta investigación exploratoria es la primera que analiza información primaria de trabajadores de Empresas B en Latinoamérica, y se centra específicamente en estudiar a los empleados jóvenes y mujeres que en ellas laboran. Este es un grupo de especial interés en este tipo de empresas por su rol como herramienta de inserción laboral, alivio de pobreza y desigualdad.

2.- Se analiza el grado de compromiso laboral de los trabajadores jóvenes y mujeres de estas empresas, una característica que, si bien no aparece explícitamente, está naturalmente vinculada con el sello distintivo de las Empresas B, las que se destacan por sus prácticas laborales, su preocupación con la comunidad y el medioambiente.

3.- Globalmente, el nivel de compromiso laboral de los trabajadores jóvenes y mujeres medido para las 16 Empresas B Latinoamericanas partícipes de este estudio fue de $79 \%$, cuatro puntos porcentuales sobre el promedio para América Latina, lo que sugiere que las prácticas laborales son globalmente valoradas por sus trabajadores.

4.- Sin embargo, a nivel de las dimensiones más vinculadas con la naturaleza de las Empresas B, los resultados son algo heterogéneos. La dimensión "Marca" es muy bien reconocida por sus trabajadores. En cambio, la dimensión "Básicos", sugiere que los beneficios obtenidos (monetarios y no monetarios) por los trabajadores generan bajos niveles de satisfacción.

5.- Los resultados permiten sugerir algunas recomendaciones para las Empresas B en Latinoamérica. En primer lugar, avanzar en mejorar la forma en que los trabajadores sienten que son partícipes de los beneficios, recompensas y reconocimientos. La segunda recomendación se refiere a intentar mejorar los niveles de comunicación interna con sus trabajadores.

\section{AGRADECIMIENTOS}

Los datos de esta investigación fueron parte de la convocatoria desarrollada por Academia B/Sistema B denominado "Caracterización y medición del impacto de las Empresas B en la inclusión de jóvenes y mujeres en América Latina" financiado con el apoyo de International Development Research Centre (IDRC) de Canadá. 


\section{REFERENCIAS}

Albdour, A. y Altarawneh, I., Employee engagement and organizational commitment: Evidence from Jordan, International Journal of Business, 19(2), 192-212 (2014)

Ali, I., Rehman, K.U., y otros tres autores, Corporate social responsibility influences, employee commitment and organizational performance, African journal of Business Management, 4(13), 2796-2801 (2010)

Aon-Hewitt, A. Tendencias Globales del Compromiso de los Empleados 2015, http://www.aon.com (2016)

Aon-Hewitt, A. Tendencias Globales del Compromiso de los Empleados 2016, http://www.aon.com (2017)

Aon-Hewitt, A. Tendencias Globales del Compromiso de los Empleados 2017, http://www.aon.com (2018)

Asmus, K., Finding the Benefit in a New Administration: A Uniform B Corporation Legislation, J. Corp. L., 43,375 (2017)

B Lab., B Corp Index Report 2012-Draft, https://sites.duke.edu (2012)

Brammer, S., Millington, A., y Rayton, B., The contribution of corporate social responsibility to organizational commitment, The International Journal of Human Resource Management, 18(10), 1701-1719 (2007)

Collier, J., y Esteban, R., Corporate social responsibility and employee commitment, Business ethics: a European review, 16(1), 19-33 (2007)

Conger, M., McMullen, J., Bergman, B., y Jeffrey Y., Category membership, identity control, and the reevaluation of prosocial opportunities, Journal of Business Venturing, 33, 179-206 (2018)

da Costa, S., Páez, D., y otros tres autores, Factors favoring innovation in organizations: An integration of meta-analyses, https://doi.org/10.1016/j.rpto.2014.06.006, Revista de Psicología del Trabajo y de las Organizaciones, 30(2), 67-74 (2014)

Fleming, J.H., y Asplund, J., Human sigma, New York, Gallup (2007)

Gehman, J. y Grimes, M., Hidden badge of honor: how contextual distinctiveness affects category promotion among certified B corporations, https://doi.org/10.5465/amj.2015.0416, Acad. Manag. J, 60(6),2294-2320 (2017)

Grimes, M.G., Gehman, J., y Cao, K., Positively deviant: Identity work through B Corporation certification, Journal of Business Venturing, 33(2), 130-148 (2018)

Martínez-Rodríguez, F. y Carmona, G., Test de factores socio personales para la inserción laboral de los jóvenes: un instrumento para la evaluación y la formación Socio personal, Estudios Sobre Educación, 18,115-138 (2010)

Maslach, C., Schaufeli, W.B. y Leiter, M.P., Job Burnout, Annual Review of Psychology, 52, 397-422 (2001)

Mehech, C., Cordero, A y Gómez, T., Medición del compromiso laboral y su impacto en los resultados de la empresa, Seminario de Titulación, Universidad de Chile, 1-91, Santiago, Chile (2016)

Ministerio de Economía Fomento y Turismo, Boletín Informalidad en el Microemprendimiento en Chile Resultados de la EME 5, División de Política Comercial e Industrial, Unidad de Estudios, Junio (2018)

Muñoz, P., Cacciotti, G. y Cohen, B., The double-edged sword of purpose-driven behavior in sustainable venturing, Journal of Business Venturing, 33, 149-178 (2018)

Muñoz, P., Latin American B Corp Sustainability Challenges 2015, Network for Business Sustainability (NBS) Chile, 11 (2015)

Ng, E.S.W., Schweitzer, L., y Lyons, S.T., New Generation, Great Expectations: A Field Study of the Millennial Generation, http://dx.doi.org/10.1007/s10869-010-9159-4, Journal of Business and Psychology, 25, $281-292$ (2010)

Padilla, C., Arévalo, D., Bustamante, M., y Vidal, C., Responsabilidad Social Empresarial y Desempeño Financiero en la Industria del Plástico en Ecuador, Información Tecnológica, 28(4), 93-102 (2017)

Pippin, S. y Weber, J., Benefit corporations and B corporations: new opportunities for accountants, The CPA Journal, 86(8), 54-57 (2016)

Salanova, M. y Schaufeli, W., El engagement de los empleados: Un reto emergente para la dirección de los recursos humanos, Estudios Financieros, 261, 106-137 (2004)

Schaufeli, W.B., y Bakker, A.B., Defining and measuring work engagement: Bringing clarity to the concept, Work engagement: a handbook of essential theory and research, Psychology Press, 10-24 (2010)

Sharma, G., Beveridge, A., y Haigh, N., A configural framework of practice change for B corporations, Journal of Business Venturing, 33, 207-224 (2018)

Shuck, B., y Wollard, K., Employee engagement and HRD: A seminal review of the foundations, Human Resource Development Review, 9(1), 89-110 (2010)

Sinval, J., Marqués-Pinto, A., Queirós, C., y Marôco, J., Work Engagement among Rescue Workers: Psychometric Properties of the Portuguese UWES, Frontiers in Psychology, 8, 1-18 (2018)

United Nations Development Programme (UNDP), Human Development Report 2016 Human Development for Everyone, UN Plaza, New York, NY 10017 EEUU (2016)

Wilburn, K., y Wilburn, R., The double bottom line: Profit and social, https://doi.org/10.1016/j.bushor.2013.10.001, Business Horizons, 57(1), 11-20 (2014) 